\title{
Estudo do mecanismo de corrosão por pites em água do mar de aços inoxidáveis supermartensíticos microligados com $\mathrm{Nb}$ e $\mathrm{Ti}$
}

\author{
Study of pitting corrosion mechanism of supermartensitic stainless \\ steels microalloyed with $\mathrm{Nb}$ and $\mathrm{Ti}$ in sea water
}

\section{Carlos Alberto Picon \\ Faculdade de Engenharia de llha Solteira FEIS-UNESP, Ilha Solteira - SP E-mail: capicone@dfq.feis.unesp.br}

\section{Frederico Augusto Pires Fernandes}

Escola de Engenharia de São Carlos EESC-USP, São Carlos - SP E-mail:codoico@gmail.com

\section{Germano Tremiliosi-Filho \\ Instituto de Química de São Carlos IQSC-USP, São Carlos - SP \\ E-mail:germano@iqsc.usp.br}

\author{
Cesar Augusto D. Rodrigues \\ Instituto de Química de São Carlos \\ IQSC-USP, São Carlos - SP \\ E-mail: cesaraug@sc.usp.br
}

\section{Luiz Carlos Casteletti}

Escola de Engenharia de São Carlos EESC-USP, São Carlos - SP E-mail: castelet@sc.usp.br

\section{Resumo}

Os aços inoxidáveis supermartensíticos (SMSS) são usados em ambientes agressivos devido à sua boa soldabilidade, boas propriedades mecânicas em temperaturas elevadas e superior resistência à corrosão sob tensão. Aplicações na exploração de petróleo demandam superior combinação de propriedades e os aços inoxidáveis duplex e superduplex têm sido aplicados nessa área, a despeito de seus custos elevados. Os SMSS consistem numa alternativa técnica e econômica ao uso daqueles aços. Nesse trabalho, adições de $\mathrm{Nb}$ e Ti foram realizadas com o intuito de minimizar o efeito de sensitização, promover o refino de grãos e foram estudados os aspectos microestruturais e a resistência à corrosão por pites em água do mar. A formação e a evolução dos pites foram acompanhadas por ensaios de corrosão, microscopia óptica e eletrônica, focalizando suas morfologias. O aço com Ti apresentou o melhor desempenho quanto à corrosão, com o maior potencial de corrosão e menor potencial de pite entre os aços em estudo. $\mathrm{O}$ aço com $\mathrm{Nb}$, apesar de apresentar potencial de corrosão superior ao do aço sem adição, teve um potencial de pite inferior ao do mesmo.

Palavras-chave: Corrosão, pites, aço inoxidável supermartensítico, microligado.

\begin{abstract}
Supermartensitic stainless steel (SMSS) is increasingly used in harsh environments due to its good weldability and mechanical properties at higher temperatures and high resistance to corrosion under stress. Applications in oil exploration demand a superior combination of properties and duplex and superduplex stainless steels have been widely applied in this area, despite their high costs. SMSS provides a technical and economical alternative for these steels. In this research, additions of $\mathrm{Nb}$ and $\mathrm{Ti}$ were made in order to minimize the sensitization effect and to promote grain refinement, studying the microstructural aspects and the pitting corrosion resistance in seawater. Pitting formation and evolution were accompanied by corrosion testing, optical and electronic microscopy. The Ti alloyed steel showed the best corrosion performance, with the greatest corrosion potential and the lowest pitting potential. The steel with $\mathrm{Nb}$ addition presented a higher corrosion potential than that of the steel without additions but had a lower pitting potential.
\end{abstract}

Keywords: Corrosion, pitting, supermartensitic stainless steel, micro-alloyed. 


\section{Introdução}

Os aços inoxidáveis supermartensíticos (Supermartensitic Stainless Steels-SMSS) exibem propriedades mecânicas e resistência à corrosão superiores às dos aços inoxidáveis martensíticos convencionais. Esses aços são muito utilizados em componentes de equipamentos nas indústrias químicas, petroquímicas e do petróleo, devido, também, a sua boa ductilidade relativa aliada ao seu menor custo (Rodrigues et al., 2007; Griffiths et al., 2004; Srinivasan; Sharkawy; Dietzel, 2004; Srinivasan; Sharkawy; Dietzel, 2004).

O processo de corrosão em água do mar profunda é caracterizado por baixas temperaturas, alta concentração de cloreto, baixa quantidade de oxigênio, presença de $\mathrm{CO}_{2}$ e $\mathrm{H}_{2} \mathrm{~S}$, microorganismos e alta concentração de sais dissolvidos. Entre os tipos de corrosão, a formação de pites é muito comum em água do mar e a mais difícil de se controlar (Anselmo et al., 2006).

Muitos metais sofrem corrosão por pites em soluções com alta concentração de cloretos, entre eles estão incluídos os aços inoxidáveis, ferro, níquel, cobre, magnésio, zircônio, estanho, cádmio, alumínio e suas ligas, etc. Alguns metais são resistentes ao pite induzido por cloretos, tais como o titânio, cromo e tântalo, porém não são imunes a ele (Isaacs et al.,1990; Pistorius; Burstein, 1992).

A corrosão por pites é um tipo de ataque muito localizado, onde a destruição é confinada a pequenas áreas, da ordem de milímetros quadrados ou menos, resultando em pequenos furos que penetram o metal, enquanto que as outras partes de sua superfície permanecem passivas (Galvele, 1978). O aparecimento do pite está relacionado à presença de certos ânions agressivos no meio. O mesmo metal pode mostrar diferentes potenciais de pite em diferentes ânions, mas, em geral, o íon cloreto é um dos mais agressivos de todos, desde que ele acarreta um baixo potencial de pite, sendo, também, o mais abundante na natureza (Galvele, 1983). Em soluções de alta concentração de cloretos, o pite é caracterizado por um potencial mínimo, chamado de potencial de pite. Abaixo desse potencial, o metal permanece passivo e, acima dele, o pite é observado, sendo este um critério usado para sua detecção, embora um exame detalhado da região passiva mostre que a corrente de passivação é mais ruidosa em soluções de cloreto do que em soluções em que este íon esteja ausente. Os pites no aço inoxidável geralmente são afastados entre si e a maior parte da superfície é passiva. Entretanto a velocidade de propagação do pite é muito rápida (Isaacs et al.,1990; Pistorius; Burstein, 1992).

O mecanismo de formação dos pites é de difícil detecção devido ao pequeno tamanho que eles apresentam, requerendo um tempo razoável para a sua visualização, ocorrendo em locais de defeitos que podem ter sido originados pelo rompimento do filme protetor sobre a superfície do metal, por descontinuidades mecânicas e ainda por heterogeneidades microestruturais (Dexter, 1987). Nesse período de tempo, necessário para a formação e crescimento dos pites, é suposto que várias reações anódicas e catódicas ocorram, ocasionando a dissolução do metal (Fontana, 1986).

O potencial de pite é uma função da composição do meio, da concentração do íon agressivo, da temperatura, da composição da liga, do tratamento superficial, etc.

Esse trabalho tem como objetivos analisar o mecanismo da formação e a evolução dos pites sobre a superfície de três aços supermartensíticos: microligados com $\mathrm{Nb}$ (SMSS+Nb) ou $\mathrm{Ti}$ (SMSS+Ti) e não microligado (SMSS), por meio de informações obtidas das curvas de polarização potenciodinâmicas cíclicas, microscopia ótica, microscopia eletrônica de varredura (MEV) e energia dispersiva de raios $\mathrm{X}$ (EDX).

\section{Material e métodos}

Os aços aqui estudados foram, inicialmente, solubilizados $\left(1000^{\circ} \mathrm{C} / 45 \mathrm{~min}\right)$ e temperados em água, em seguida revenidos a $570^{\circ} \mathrm{C} / 2 \mathrm{~h}$ e resfriados ao ar. Para os testes de corrosão, os materiais foram preparados por meio de técnicas convencionais metalográficas, até obtenção de uma superfície isenta de riscos A célula eletroquímica utilizada, para a obtenção das curvas de polarização potenciodinâmicas, consta de um eletrodo de referência de calomelano saturado (ECS) e de um eletrodo auxiliar de platina. O eletrólito empregado foi água do mar natural (PH 8,0) obtida em alto mar da praia da Boa Viagem em Recife-PE. Para a realização dos testes, foi empregado um potenciostato modelo Autolab-VGSTAT-302. A velocidade de varredura usada foi de $1 \mathrm{mV} / \mathrm{s}$.

Para as observações metalográficas foi empregado um microscópio óptico Zeis modelo Axiotech. As medidas de microdureza Vickers foram efetuadas em um equipamento digital empregando carga de 100gf/10s. A microscopia eletrônica foi realizada em um Microscópio Eletrônico de Varredura (MEV), modelo 440 LEO com filamento de tungstênio, no modo de operação com elétrons secundários. A Tabela 1 apresenta as composições químicas nominais em porcentagem em peso dos aços estudados.

\section{Resultados e discussão}

A Figura 1 apresenta as microestruturas ópticas dos três aços estudados, na condição revenida.

Para o aço sem adições, verificase uma estrutura martensitica do tipo "lath”. A adição de nióbio ou titânio produziu um refino considerável de grão.

As curvas de polarização potenciodinâmica anódicas representativas dos três aços estão mostradas na Figura 2.

Verifica-se que os três aços supermartensíticos apresentaram a passivação com posterior indicação da formação de pites (Figura 2).

Na Tabela 2, são apresentados os parâmetros coletados das curvas de polarização potenciodinâmicas e os valores de microdureza, para os aços em questão. 
Carlos Alberto Picon et al.

Tabela 1 - Composição química nominal em porcentagem em peso dos aços estudados.

\begin{tabular}{c|c|c|c|c|c|c|c|c|c|c}
\hline & \multicolumn{10}{|c}{ Elementos } \\
\hline Liga & $\mathbf{C}$ & $\mathbf{C r}$ & $\mathbf{N i}$ & $\mathbf{M o}$ & $\mathbf{M n}$ & $\mathbf{S i}$ & $\mathbf{S}$ & $\mathbf{P}$ & $\mathbf{N b}$ & $\mathbf{T i}$ \\
\hline SMSS & 0,013 & 12,50 & 5,05 & 2,12 & 0,30 & 0,18 & 0,0014 & 0,005 & - & - \\
\hline SMSS+Nb & 0,019 & 12,50 & 5,36 & 2,10 & 0,31 & 0,21 & 0,0010 & 0,005 & 0,20 & - \\
\hline SMSS+Ti & 0,017 & 12,50 & 5,39 & 2,09 & 0,30 & 0,30 & 0,0010 & 0,005 & - & 0,13 \\
\hline
\end{tabular}
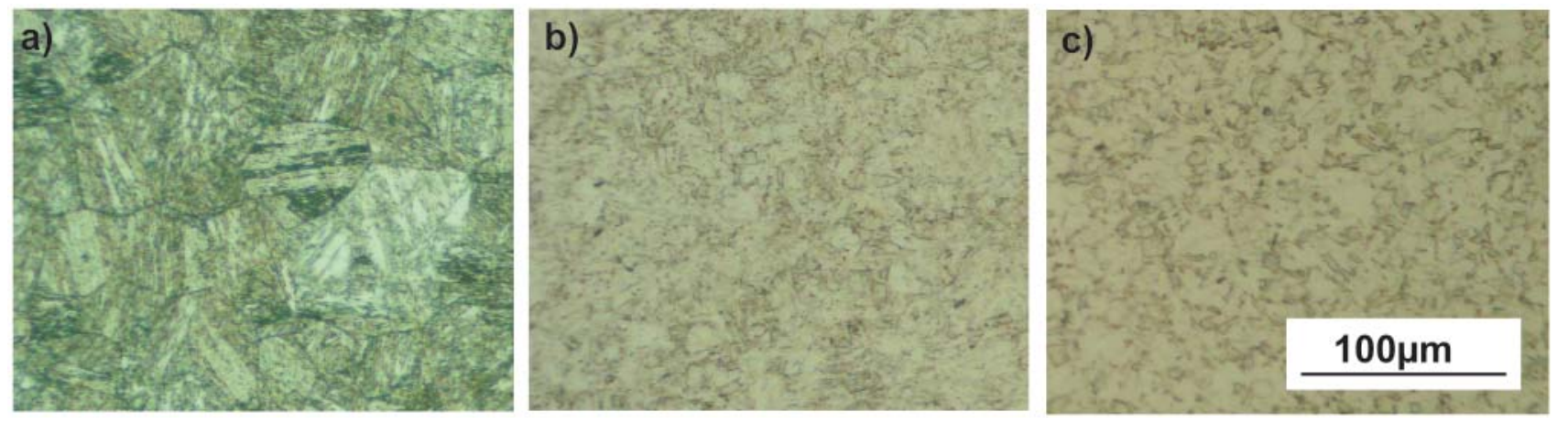

Figura 1 - Micrografias ópticas dos aços estudados: a) SMSS; b) SMSS+Nb; c) SMSS+Ti.

Pode ser observado que os parâmetros, tais como potencial e corrente de corrosão e pite, gerados da análise dessas curvas para os três aços, de um modo geral, mostram que, em água do mar natural, o aço microligado com Ti apresentou o melhor desempenho à corrosão, pois, além da formação do pite ser posterior aos outros dois aços, a corrente de corrosão é muito mais baixa, conforme Tabela 2.

A Figura 3 mostra micrografias ópticas das superfícies dos aços após exposição em água do mar natural.

Nota-se que, ao redor dos pites, no aço sem adição, ocorrem a presença de pites secundários e maior deterioração, conforme é mostrado na Figura 3, nas mesmas condições, para o levantamento das três curvas. Para os aços microligados, são observados pites com maior definição e, preferencialmente, com geometria circular.

Em relação à microdureza dos aços (Tabela 2), observa-se que a adição de nióbio e titânio promove um pequeno aumento nessa propriedade, quando

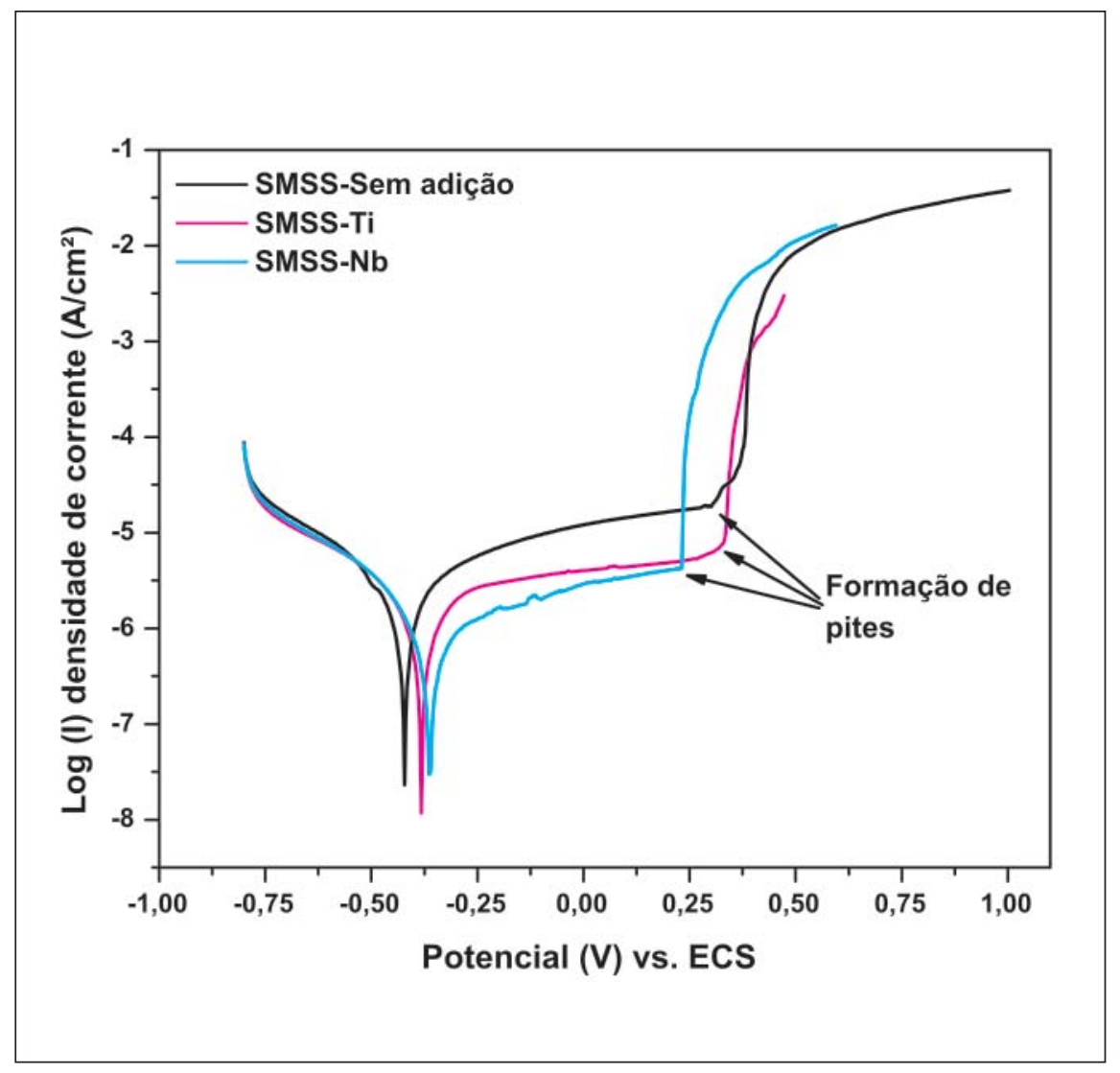

Figura 2 - Curvas de polarização potenciodinâmicas anódicas dos aços estudados. 
Estudo do mecanismo de corrosão por pites em água do mar de aços inoxidáveis supermartensíticos...

Tabela 2 - Resultados dos parâmetros das curvas de corrosão e dureza dos aços estudados.

\begin{tabular}{c|c|c|c}
\hline \multirow{2}{*}{ Parâmetro } & \multicolumn{3}{|c}{ Amostra } \\
\cline { 2 - 4 } & SMSS & SMSS+Nb & SMSS+Ti \\
\hline Potencial de corrosão, mV & -422 & -364 & -382 \\
\hline Corrente corrosão, $\mathbf{n A} / \mathbf{c m}^{2}$ & 23,1 & 29,7 & 11,7 \\
\hline Potencial de pite, $\mathbf{m V}$ & 308 & 231 & 326 \\
\hline Corrente de pite, $\boldsymbol{\mu A} / \mathbf{c m}^{2}$ & 20,67 & 4,23 & 7,46 \\
\hline Microdureza, HV & $277(5)$ & $292(5)$ & $295(4)$ \\
\hline
\end{tabular}

comparada ao aço sem adição de microligante.

A microscopia eletrônica de varredura, que ilustra os pites sobre a superfície dos três aços (Figura 4), originados nas regiões indicadas pelas setas nas curvas de polarização (Figura 2), apresenta formas e diâmetros semelhantes, exceto para o aço sem adição, o que resultou na formação de irregularidades e pites secundários.
A Tabela 3 apresenta os valores obtidos via análises de Energia Dispersiva de Raios X (EDX) em região fora dos pites e nos interiores dos mesmos.

Análises realizadas nos interiores dos pites indicaram uma maior concentração de Cr, Ni e Mo em comparação com a composição-base, no aço microligado com Nb. Tal fato deve-se, provavelmente, à reação autocatalítica que ocorre em um pite de corrosão, com a evolução interna de hidrogênio e a migração dos íons de cloreto para o interior do pite, a partir da solução para neutralização das cargas elétricas. Isto provoca uma alta concentração de cloretos dentro dos pites (Dexter, 1987). Tal cloreto, ao atacar o material, acarretará a retirada do mesmo do local, podendo, adicionalmente, ocorrer a formação de cloreto férrico, que é altamente agressivo, acelerando o processo. Com a retirada do ferro, ocorre, conseqüentemente, um aumento na concentração dos outros elementos. No caso do aço com Ti, o teor de Fe, em quantidade relativamente maior, deve-se ao menor ataque do mesmo.

$\mathrm{O}$ aço microligado com Nb apresentou o menor potencial de pite entre os aços estudados, conforme pode ser observado na Tabela 2. Nos ensaios realizados por microssonda eletrônica (EDX), foi verificado que a porcentagem atômica dos elementos $\mathrm{Cr}, \mathrm{Ni}$, Mo e Nb (no interior do pite) aumentou em relação à análise realizada fora do pite.

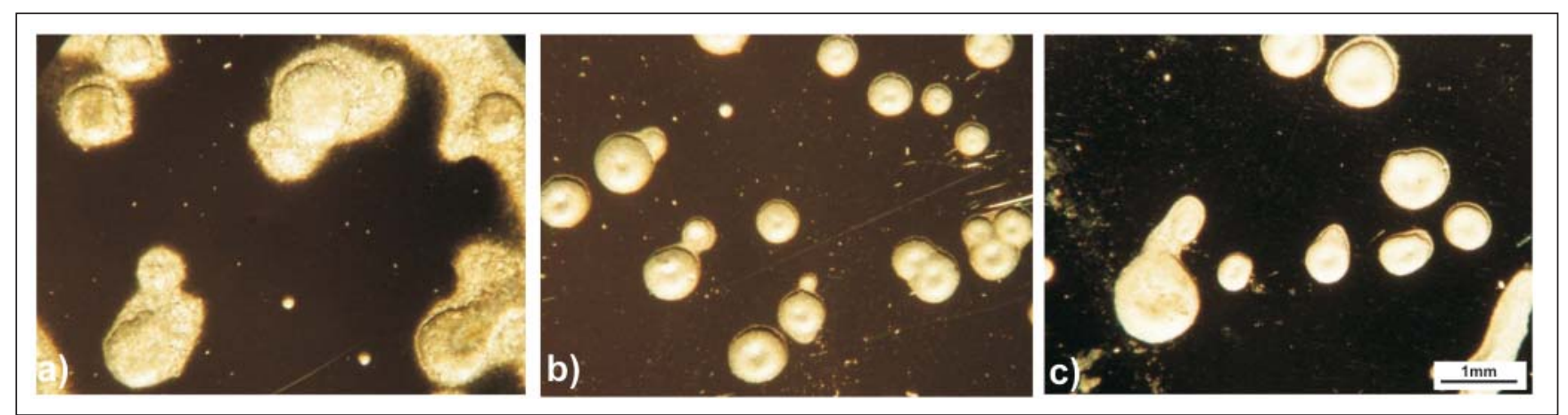

Figura 3 - Aspecto da superfície das amostras após ensaio de corrosão: a) SMSS; b) SMSS+Nb; c) SMSS+Ti.

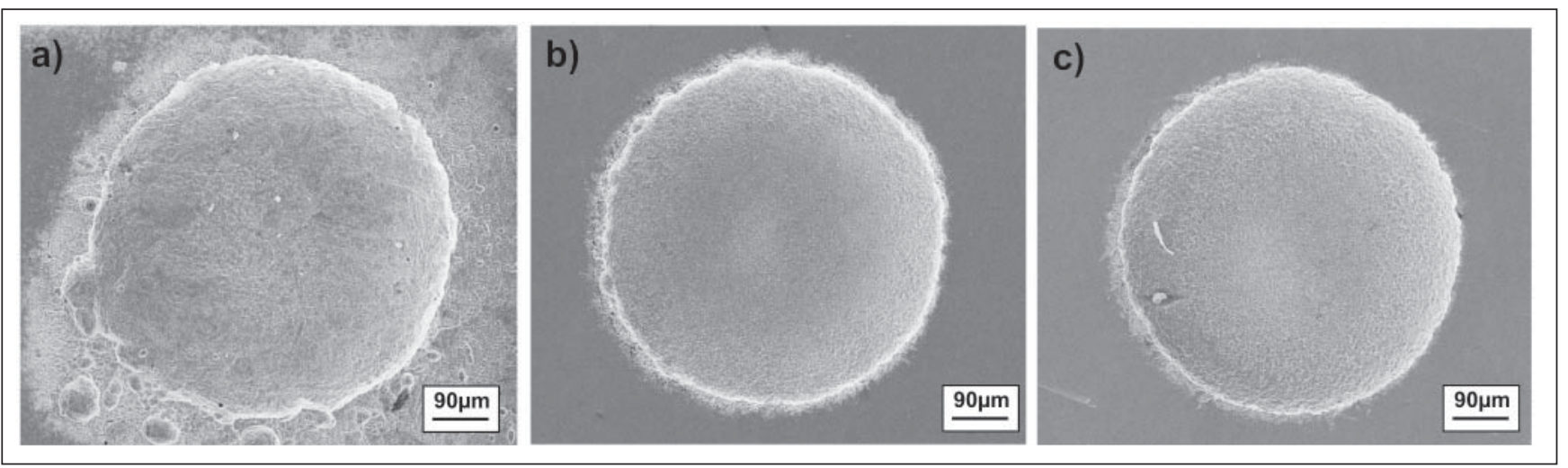

Figura 4 - Morfologia dos pites encontrados para os aços estudados: a) SMSS; b) SMSS+Nb; c) SMSS+Ti. 
Tabela 3 - Porcentagens atômicas para os aços estudados.

\begin{tabular}{c|c|c|c|c|c|c}
\hline & \multicolumn{6}{|c}{ Elementos } \\
\hline Liga & Fe & Cr & Ni & Mo & Nb & Ti \\
\hline SMSS & 80,21 & 14,11 & 4,52 & 1,17 & -- & -- \\
\hline SMSS+Nb & 80,12 & 13,94 & 4,45 & 1,29 & 0,21 & -- \\
\hline SMSS+Nb (pite) & 78,88 & 14,11 & 5,29 & 1,43 & 0,29 & -- \\
\hline SMSS+Ti & 79,22 & 14,30 & 4,98 & 1,15 & -- & 0,35 \\
\hline SMSS+Ti (pite) & 80,59 & 13,90 & 4,44 & 0,85 & -- & 0,23 \\
\hline
\end{tabular}

Já o conteúdo de Fe diminuiu. Em relação às observações anteriores, para o aço com adição de Ti, ocorreu o inverso.

\section{Conclusões}

O aços estudados apresentaram valores de microdureza semelhantes e estrutura martensitica do tipo “lath”, porém os materiais com adição de Nb ou Ti exibiram considerável refino de grão.

O aço com Ti apresentou o melhor desempenho quanto à corrosão, com o maior potencial de corrosão e o menor potencial de pite entre os aços em estudo. O aço com Nb, apesar de apresentar potencial de corrosão superior ao do aço sem adição, teve um potencial de pite inferior ao do mesmo.

\section{Agradecimentos}

Os autores agradecem a CAPES pela bolsa de estudo concedida a Frederico Augusto Pires Fernandes e ao CNPq (proc. 150799/2008-0).

\section{Referências bibliográficas}

ANSELMO, N. et al. Corrosion behavior of supermartensitic stainless steel in aerated and CO2-saturated synthetic seawater. Materials Science and Engineering A, v.428, p. 73-79, 2006.

DEXTER, S.C. Localized corrosion. In Corrosion, v. 13, ASM International, p.104, 1987.

FONTANA, M.G. Corrosion engineering. McGraw-Hill Book Company, 1986. 282p.
GALVELE, J.R. Passivity of metals. In: FRANKENTHAL, R. P., KRUGER, J. (eds.). New Jersey: The Electrochemical Society, Inc., 1978. p.285-327.

GALVELE, J.R. Pitting corrosion (J.C.Scully, ed.) Treatise on Materials Science and Technology, New York: Academic Press., 1983, v. 23, p.1-53.

GRIFFITHS, A. et al. A novel approach to characterizing the mechanical properties of supermartensitic $13 \mathrm{Cr}$ stainless steel welds. Materials Science and Engineering A, v.384, p. 83-91, 2004.

ISAACS, H. S. et al. Advances in localized corrosion. NACE. Houston, Texas, p.393, 1990.

PISTORIUS, P. C., BURSTEIN, G. T. Phil. Trans. Roy. SOC. Lond. series A, 341, 531, 1992.

RODRIGUES, C.A.D. et al. Titanium and molybdenum content in supermartensitic stainless steel. Materials Science and Engineering A, v.460-461, p. 149-152, 2007.

SRINIVASAN, P.B., SHARKAWY, S.W.,DIETZEL, W. Environmental cracking behavior of submerged arc-welded supermartensitic stainless steel weldments. Journal of Materials Engineering and Performance, v.13, n. 2, p. 232-236, 2004.

SRINIVASAN, P.B., SHARKAWY, S.W., DIETZEL, W. Hydrogen assisted stress-cracking behaviour of electron beam welded supermartensitic stainless steel weldments. Materials Science and Engineering A, v.385, p. 6-12, 2004.

Artigo recebido em 08/06/2009 e aprovado em 19/01/2010.

$$
\begin{aligned}
& \text { A REM tem novo endereço: } \\
& \text { FUNDAÇÃO GORCEIX - REM } \\
& \text { Rua Carlos Walter Marinho Campos, } 57 \text { - Bairro: Vila Itacolomy } \\
& \text { 35400-000 - Ouro Preto - MG } \\
& \begin{array}{ll}
\text { (31) } 3551-4730 & \text { (31) } 3559-7408
\end{array}
\end{aligned}
$$

